Fukushima, M. and Takeda, M.

Osaka J. Math.

21 (1984), 311-326

\title{
A TRANSFORMATION OF A SYMMETRIC MARKOV PROCESS AND THE DONSKER-VARADHAN THEORY
}

\author{
Masatoshi FUKUSHIMA and Masayoshi TAKEDA
}

(Received May 10, 1983)

\section{Introduction}

In obtaining the lower bound in the celebrated law of large deviation of the occupation distribution for the one dimensional Brownian motion, Donsker and Varadhan [3] performed a transformation of the absorbing Brownian motion on an interval $(a, b)$ by a drift $b=(\log \rho)^{\prime}$, or equivalently, by a multiplicative functional

$$
\frac{\rho\left(B_{t}\right)}{\rho\left(B_{0}\right)} \exp \left(-\int_{0}^{t} \frac{\rho^{\prime \prime}}{2 \rho}\left(B_{s}\right) d s\right) I_{\{t<\zeta\}}
$$

into a conservative process on $(a, b)$ with invariant probability measure $\rho^{2} d x$, to which the ergodic theorem was well applied. Here $\rho^{2}$ is assumed to be a probability density $C^{2}$-function on $R^{1}$, positive inside $(a, b)$ and vanishing outside.

We show in this paper that their method works for any symmetric Hunt process corresponding to a regular and irreducible Dirichlet form. In the present general case, we take function $\rho$ from the range of the resolvent. In order to prove the conservativeness of transformed process, we make full use of an explicit expression of the transformed Dirichlet form, while the Feller test of non-explosion was available in the special case of [3].

We consider a locally compact separable metric space $X$ and a positive Radon measure $m$ on $X$ such that $\operatorname{Supp}[m]=X$. The inner product in real $L^{2}$ space $L^{2}(X ; m)$ is denoted by $($,$) and C_{0}(X)$ stands for the space of continuous functions on $X$ with compact support. Let $\boldsymbol{M}=\left(X_{t}, \boldsymbol{P}_{x}, \zeta\right)$ be a Hunt process on $X$ which is $m$-symmetric in the sense that the transition function $P_{t}$ satisfies $\left(P_{t} f, g\right)=\left(f, P_{t} g\right), f, g \in C_{0}(X)$. Then the Dirichlet form $E$ of $M$ can be defined by $F=D[E]=D(\sqrt{-A}), E(u, v)=(\sqrt{-A u}, \sqrt{-A v})$ where $A$ is the infinitesmal generator of the semigroup on $L^{2}(X ; m)$ determined by $P_{t}$. We always assume that $E$ is regular: $F \cap C_{0}(X)$ is dense both in $F$ and in $C_{0}(X)$,

In $\S 2$, we derive the Beurling-Deny formula 


$$
\begin{gathered}
E(u, v)=\frac{1}{2} \int_{X} d_{\mu_{<u, v>}^{c}}^{c}+\int_{X x X-d}(\tilde{u}(x)-\tilde{u}(y))(\tilde{v}(x)-\tilde{v}(y)) J(d x, d y)+\int_{X} \tilde{u} \tilde{v} k \\
u, v \in F,
\end{gathered}
$$

together with the derivation property of the local energy measure $\stackrel{c}{\mu}_{<u, v>}$ due to Le Jan ([11]). Our approach is more comprehensive than [6] and [11], and indeed we first decompose the martingale part $M_{t}^{[u]}$ of the additive functional $\tilde{u}\left(X_{t}\right)-\tilde{u}\left(X_{0}\right)$ and then compute the energy of each term.

In $\S 3$, we perform the above mentioned transformation of $\boldsymbol{M}$ to get a $\rho^{2} m$-symmetric process $\tilde{\boldsymbol{M}}$. Using the fact that the multiplicative functional involved is a solution of a Doleans-Dade equation related to the martingale $M^{[\rho]}$, the Dirichlet form $\widetilde{E}$ of $\tilde{\boldsymbol{M}}$ is shown to have the expression

$$
\widetilde{E}(u, v)=\frac{1}{2} \int_{X} \rho^{2} d \tilde{\mu}_{<u, v>}+\int_{X x X-d} \tilde{u}((x)-\tilde{u}(y))(\tilde{v}(x)-\tilde{v}(y)) \rho(x) \rho(y) J(d x, d y) .
$$

We can then conclude that $1 \in D[\widetilde{E}]$ and $\widetilde{E}(1,1)=0$, which simply means the conservativeness of $\tilde{\boldsymbol{M}}$.

In $\S 4$, we assume that there exist relatively compact open sets $G_{n}$ increasing to $X$ and the part of the Dirichlet form $E$ to each set $G_{n}$ is irreducible. Let

$$
L(t, A)=\frac{1}{t} \int_{0}^{t} I_{A}\left(X_{s}\right) d s, \quad t<\zeta,
$$

then the set function $L(t, \cdot)$ called the occupation distribution is an element of the space $\mathscr{M}$ of probability measures on $X$. We then have just as in [3] the estimate

$$
\varliminf_{t \rightarrow \infty} \frac{1}{t} \log \boldsymbol{E}_{x}\left(e^{-t \Phi(L(t, \cdot))} ; t<\zeta\right) \geqq-\inf _{\rho \in V}\left[\Phi\left(\rho^{2}\right)+E(\rho, \rho)\right],
$$

where $V=\left\{\rho \in F ; \rho^{2} m \in \mathscr{M}\right.$, Supp $\left[\rho^{2} m\right]$ is compact $\}$ and $\Phi$ is any functional on $\mathscr{M}$ such that $\Phi\left(\mu_{n}\right) \rightarrow \Phi(\mu)$ whenever $\mu_{n} \in \mathscr{M}$ converge weakly to $\mu$ and the support of $\mu_{n}$ is contained in a common compact set. The above inequality holds for every $x \in X$ except possibly on a set of zero capacity which is independent of $\Phi$.

Since any regular Dirichlet form admits an associated symmetric Hunt process, we may say that the present lower estimate is an intrinsic property of Dirichlet form which is regular and irreducible. These two conditions on Dirichlet form are very mild and directly verifiable. We do not assume the Feller property nor the absolute continuity of the associated transition function, although the transition function is always symmetric in our setting.

For instance, consider locally integrable functions $a_{i, j}(x), 1 \leqq i, j \leqq d$, on 
the $d$-space $R^{d}$ satisfying $a_{i, j}=a_{j, i}$ and $\inf _{x \in K,|\xi|=1} \sum_{i, j=1}^{d} a_{i, j}(x) \xi_{i} \xi_{j}>0$ for any compact set $K$, then the form

$$
E(u, v)=\sum_{i, j=1} \int_{R^{d}} \frac{\partial u}{\partial x_{i}}(x) \frac{\partial v}{\partial x_{j}}(x) a_{i, j}(x) d x, \quad u, v \in C_{0}^{1}\left(R^{d}\right),
$$

is closable on $L^{2}\left(R^{d}\right)$ and the closure $\bar{E}$ becomes a regular and irreducible Dirichlet form on $L^{2}\left(R^{d}\right)$. Here $C_{0}^{1}\left(R^{d}\right)$ is the space of $C^{1}$-functions in $C_{0}\left(R^{d}\right)$. More generally, we may replace $a_{i, j}(x) d x$ by a Radon measure $\nu_{i, j}$ such that $\nu_{i, j}=\nu_{j, i}$ and $\inf _{|\xi|=1} \sum_{i, j=1}^{d} \nu_{i, j}(K) \xi_{i} \xi_{j} \geqq \delta_{K}|K|$ for any compact $K$ where $\delta_{K}$ is positive constant and $|K|$ is the Lebesgue measure of $K$. Under the closability assumption of the associated form $E$ on $C_{0}^{1}\left(R^{d}\right)$, we have a same kind of form $\bar{E}$. We can instead start with a jumping measure of the type $J(d x, d y)=$ $N(x, d y) d x$ which makes the associated symmetric form

$$
E(u, v)=\int_{R^{d} x R^{d}-d}(u(x)-u(y))(v(x)-v(y)) N(x, d y) d x, \quad u, v \in C_{0}^{1}\left(R^{d}\right),
$$

closable on $L^{2}\left(R^{d}\right)$. In this case, it suffices to assume $\operatorname{Supp}[N(x, \cdot)]=R^{d}$ for almost all $x$ in order to obtain the Dirichlet form $\bar{E}$ possessing the required properties. In each of the above three examples, the stated lower estimate holds for the Hunt process on $R^{d}$ associated with $\bar{E}$. See [6] for closability criteria for the second and third examples. We know from the works in theory of partial differential equations due to Nash et al. that the transition function in the first example is Feller and absolutely continuous. We do not know about this for the second and third examples in general. But see Tomisaki [12] for relevant information.

Donsker and Varadhan have extended their result in [3] to wider classes of Markov processes by finding sufficient conditions on the transition functions ([4], [5]). As for the lower estimate in the case of the complete separable metrizable state space, their conditions on the transition function (Hypothesis $H_{1} \sim H_{4}$ in [5]) include the Feller property and an absolutely continuity in addition to a transitivity assumption. The intrinsic quantity appearing in their upper and lower bounds is the $I$-functional rather than the Dirichlet form, but those two characteristics have been identified when the transition function is symmetric and absolutely continuous ([4]). As for the upper estimate, we only note that the relevant statements in [3] remain true for any Markov process with Feller transition function.

\section{Decomposition of martingale additive functionals and the Beurling-Deny formula}

Let $(E, F)$ be a $C_{0}$-regular Dirichlet space and $\boldsymbol{M}=\left(X_{t}, \boldsymbol{P}_{x}, \zeta\right)_{x \in X}$ be the 
associated $m$-symmetric Hunt process, and we use the relevant notions and notations in Fukushima [6], Dellacherie-Meyer [1]. By Theorem 5.2.2 in [6], the additive functional ( $A F$ in abbriviation) $A_{t}^{[u]}=\tilde{u}\left(X_{t}\right)-\tilde{u}\left(X_{0}\right), u \in F$, has a unique decomposition

$$
A^{[u]}=M^{[u]}+N^{[u]}
$$

where $M^{[u]}$ is a square integrable martingale $A F$ of finite energy and $N^{[u]}$ is a continuous $A F$ of zero energy. We further decompose $M^{[u]}$ as

$$
M^{[u]}=\stackrel{c}{M}^{[u]}+\stackrel{d}{M}^{[u]}=\stackrel{c}{M}^{[u]}+\stackrel{j}{M}^{[u]}+\stackrel{k}{M}^{[u]}
$$

where $\stackrel{c}{M}^{[u]}$ and $\stackrel{d}{M}^{[u]}$ are continuous and purely discontinuous part of $M^{[u]}$ respectively, and $\stackrel{M}{ }^{[u]}$ and $\stackrel{M}{ }^{[u]}$ are defined by

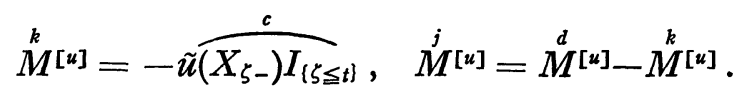

Here, for an additive functional $A$, it's compensator $\hat{A}$ is defined by $A-A^{p}$ with $A^{p}$ being the dual predictable projection of $A$ (see [1; Definition 73]). Using the co-energy $e$ of $A F$ 's, we can define three symmetric forms by

$$
\begin{aligned}
& E^{(c)}(u, v)=e\left(\stackrel{c}{M}^{[u]}, \stackrel{c}{M}^{[v]}\right) \quad E^{(j)}(u, v)=e\left(\stackrel{j}{M}^{[u]}, \stackrel{j}{M}^{[v]}\right) \\
& E^{(k)}(u, v)=2 e\left(\stackrel{M}{M}^{[u]}, \stackrel{k}{M}^{[v]}\right) .
\end{aligned}
$$

Let $(N(x, d y), H)$ be a Lévy system of Hunt process $\boldsymbol{M}$ and $\nu$ be a smooth measure corresponding to $H$. We put

$$
J(d x, d y)=\frac{1}{2} N(x, d y) \nu(d x), \quad k(d x)=N(x, \Delta) \nu(d x)
$$

and call them the jumping measure and the killing measure respectively. Since $\left\langle\dot{j}^{[u]}, \dot{j}^{[v]}\right\rangle_{t}=\left[M^{[u]}, M^{[v]}\right]_{t}^{p}=\int_{0}^{t} \int_{X}\left(\tilde{u}\left(X_{s}\right)-\tilde{u}(y)\right)\left(\tilde{v}\left(X_{s}\right)-\tilde{v}(y)\right) N(x, d y) d H_{s}$ we have

$$
E^{(j)}(u, v)=\int_{X x X-d}(\tilde{u}(x)-\tilde{u}(y))(\tilde{v}(x)-\tilde{v}(y)) J(d x, d y) .
$$

Further, since $\left\langle\stackrel{k}{M^{[u]}}, \stackrel{k}{M^{[v]}}\right\rangle_{t}=\int_{0}^{t} \tilde{u}\left(X_{s}\right) \tilde{v}\left(X_{s}\right) N\left(X_{s}, \Delta\right) d H_{s}$, we have

$$
E^{(k)}(u, v)=\int_{X} \tilde{u}(x) \tilde{v}(x) k(d x)
$$

Lemma 2.1. 1) For any $u \in F^{+}(u \in F$ and $u \geqq 0, m$-a.e. $)$ and $\alpha>0, \tilde{u} \cdot k$ is of finite energy integral and $\boldsymbol{E}_{x}\left(e^{-\infty \zeta} u\left(X_{\zeta_{-}}\right)\right)$is a quasi-continuous version of $U_{\omega}(\tilde{u} \cdot k)$. 
2) For $u \in F$

$$
\lim _{t \downarrow 0} \frac{1}{t} \boldsymbol{E}_{u^{2} m}\left(I_{(\zeta \leqq t)}\right)=\left\langle k, \tilde{u}^{2}\right\rangle
$$

Proof. 1) Since (2.2) is an orthogonal decomposition with respect to $e, \frac{1}{2} E^{(k)}(u, u)$ is dominated by $e\left(M^{[u]}, M^{[u]}\right) \leqq E(u, u)$. Therefore, by Schwarz inequality and (2.7),

$$
\int_{X}|f| \tilde{u} d k \leqq C \sqrt{E(f, f)}, \quad f \in F \cap C_{0}
$$

which means $\tilde{u} \cdot k$ is of finite energy integral. By noticing that $\boldsymbol{E}_{x}\left(e^{-\infty \zeta} \tilde{u}\left(X_{\zeta-}\right)\right)$ equals $\left.\boldsymbol{E}_{x}\left(\int_{0}^{\infty} e^{-\alpha s} \tilde{u}\left(X_{s}\right) d\left(I_{\left(X_{\zeta-\neq} \neq, \zeta \leqq s\right.}\right)^{p}\right)\right)$, we get the second assertion of 1$)$.

2) For any $h \in C_{0}^{+}$and $f \in F_{b}^{+}$,

$$
\begin{aligned}
& \boldsymbol{E}_{R_{\infty \omega h} \cdot f m}\left(I_{\{\zeta \leqq t\}}\right)=\left(h, R_{\infty}\left(f \cdot\left(1-P_{t} 1\right)\right)\right) \\
& =\boldsymbol{E}_{h m}\left(\int_{\zeta-t}^{\zeta} e^{-\infty s} \tilde{f}\left(X_{s}\right) d s ; t<\zeta\right)+\boldsymbol{E}_{h m}\left(\int_{0}^{\zeta} e^{-\infty \omega s} \tilde{f}\left(X_{s}\right) d s ; t \geqq \zeta\right) .
\end{aligned}
$$

But, since $\frac{1}{t^{2}} \boldsymbol{E}_{h m}\left(\int_{0}^{\zeta} e^{-a s} \tilde{f}\left(X_{s}\right) d s ; t \geqq \zeta\right)^{2} \leqq \frac{1}{t} \boldsymbol{E}_{h m}\left(\left(\int_{0}^{t} e^{-\alpha s} \tilde{f}\left(X_{s}\right) d s\right)^{2}\right) \cdot \frac{1}{t} \boldsymbol{P}_{h m}(t \geqq \zeta)$ $\rightarrow 0(t \rightarrow 0)$, we have

$$
\lim _{t \downarrow 0} \frac{1}{t} \boldsymbol{E}_{R_{\alpha h \cdot f m}}\left(I_{(\zeta \leqq t)}\right)=\boldsymbol{E}_{h m}\left(e^{-\alpha_{\zeta}} \tilde{f}\left(X_{\zeta-}\right)\right)=\left(h, U_{\infty}(\tilde{f} \cdot k)\right)=\left\langle k, R_{\infty} h \cdot \tilde{f}\right\rangle .
$$

In particular, $\lim _{t \downarrow 0} \frac{1}{t} \boldsymbol{E}_{\left(R_{a} h\right)^{2} m}\left(I_{\{\zeta \leqq t\}}\right)=\left\langle k,\left(R_{\omega} h\right)^{2}\right\rangle$. We can prove the relation (2.8) for general $u \in F$ in the same way as in [6; Lemma 4.5.2]. q.e.d.

Denote by $\mu_{d u\rangle}, \stackrel{c}{\mu}\langle u\rangle_{d}$, and $\stackrel{d}{\mu}_{\langle u\rangle}$ the smooth measure of $\left\langle M^{[u]}\right\rangle,\left\langle\dot{c}^{[u]}\right\rangle$ and $\left\langle\stackrel{d}{M}^{[u]}\right\rangle$ respectively, $u \in F$.

Lemma 2.2. If $u \in F$ is constant on an open set $G$, then $\stackrel{c}{\mu}\langle u\rangle_{\langle\text {on }}=0$.

Proof. Define $B_{t}^{(n)}=\sum_{k=1}^{n}\left(\tilde{u}\left(X_{\frac{k}{n} t}\right)-\tilde{u}\left(X_{\frac{k-1}{n} t}\right)\right)^{2}$, then $B_{t}^{(n)}$ equals zero on $t<\tau_{G}=\inf \left\{t ; X_{t} \notin G\right\}$. On the other hand, $\lim _{n \rightarrow \infty} B_{t}^{(n)}=\left[M^{[u]}\right]_{t}, t<\tau_{G}, \boldsymbol{P}_{m}$-a.e., because of the property of $N^{[u]}$ that $\sum_{k=1}^{n}\left(N_{\frac{k}{n} t}^{[u]}-N_{\frac{k-1}{n} t}^{[u]}\right)^{2}$ tends to zero in $L^{1}\left(\boldsymbol{P}_{m}\right)$. Since $\left[M^{[u]}\right]_{t}=\left\langle\dot{M}^{[}[u]\right\rangle_{t}+\sum_{0<^{s} \geqq t}\left(\tilde{u}\left(X_{s}\right)-\tilde{u}\left(X_{s-}^{n}\right)\right)^{2}$,

$$
\left\langle\stackrel{c}{M}^{[u]}\right\rangle_{t}=0, \quad t \leqq \tau_{G}, \quad \boldsymbol{P}_{m} \text {-a.e. }
$$

which implies the lemma by virtue of [6; Lemma 5.1.5].

q.e.d. 
Lemma 2.3. For any $u, v \in F \cap C_{0}^{+}$such that $\operatorname{Supp}[u] \cap \operatorname{Supp}[v]=\phi$,

$$
\int_{X x X-d} u(x) v(x) J(d x, d y)=-\frac{1}{2} E(u, v) .
$$

Proof. Consider a relatively compact open set satisfying $\operatorname{Supp}[u] \subset G \subset$ $\bar{G} \subset(\operatorname{Supp}[v])^{c}$. We put $N f(x)=\int_{X} f(y) N(x, d y)$. Since

$$
e\left(\stackrel{j}{M}^{[|f|]}, \stackrel{j}{M}^{[v]}\right)=-\frac{1}{2}\left(\int_{X} v \cdot N|f| \cdot \nu+\int_{X}|f| \cdot N v \cdot \nu\right) \quad \text { for } f \in F_{G} \cap C_{0},
$$

we have

$$
\int_{X}|f| \cdot N v \cdot \nu \leqq 2 e\left(M^{j}[|f|]\right)^{1 / 2} \cdot e\left(M^{j}[v]\right)^{1 / 2} \leqq C \sqrt{E(f, f)},
$$

and hence $I_{G} \cdot N v \cdot \nu$ is of finite energy integral with respect to $E_{G}$. We then have $H_{\alpha}^{X-G} v(x)=\boldsymbol{E}_{x}\left(\int_{0}^{\tau_{G}} e^{-\alpha s} N v\left(X_{s}\right) d H_{s}\right)=U_{a}^{G}(N v \cdot \nu)$ on $G$ and $H_{a}^{X-G} v=$ $U^{G}(N v \cdot \nu)+v$. It follows from $E_{a}\left(H_{a}^{X-G} v, u\right)=0$ that

$$
E\left(U_{a}^{G}(N v \cdot \nu), u\right)=2 \int_{X x X-d} u(x) v(y) J(d x, d y)=-E(u, v) . \quad \text { q.e.d. }
$$

Theorem 2.1 (Representation of the Dirichlet form E). It holds that

$$
E(u, v)=E^{(c)}(u, v)+E^{(j)}(u, v)+E^{(k)}(u, v), \quad u, v \in F .
$$

$E^{(c)}$ has the local property; $E^{(c)}(u, v)=0$ whenever $u$ is constant on the support of $v . E^{(j)}$ and $E^{(k)}$ are expressed by (2.6) and (2.7) respectively. Moreover the measure $J$ is symmetric.

Proof. By virtue of Lemma 2.1,

$$
\begin{aligned}
e\left(M^{[u]}\right) & =\lim _{t \downarrow 0} \frac{1}{2 t} \boldsymbol{E}_{m}\left(\left(\tilde{u}\left(X_{t}\right)-\tilde{u}\left(X_{0}\right)\right)^{2}\right) \\
& =\lim _{t \downarrow 0} \frac{1}{t}\left\{\left(u, u-P_{t} u\right)-\frac{1}{2}\left(u^{2}, 1-P_{t} 1\right)\right\} \\
& =E(u, u)-\frac{1}{2} \int_{X} \tilde{u}^{2} d k,
\end{aligned}
$$

and we have (2.11) in view of (2.2) and (2.4). Other assertions follow from Lemma 2.2 and Lemma 2.3.

q.e.d.

This theorem says that formula (2.11) is nothing but the Beurling-Deny formula ([6]), and moreover the symmetric measure $J$ admits a specific expression (2.5). 



in the same way.

Lemma 2.4. It holds that

$$
d_{\mu}^{c} \mu_{\left\langle u^{2}, v\right\rangle}=2 u d \stackrel{c}{\mu}_{\langle u, v\rangle} \quad \text { for } u, v \in F \cap C_{0}
$$

Proof. By the same method as in [6; Lemma 5.4.1] we have

$$
\begin{aligned}
& \int_{X} f d_{\mu_{\left\langle u^{2}, v\right\rangle}-2 \int_{X} f u d \mu_{\langle u, v\rangle}} \\
& \quad=2 \int_{X x X-d}(u(x)-u(y))^{2}(v(x)-v(y)) f(x) J(d x, d y)-\int_{X} f u^{2} v k .
\end{aligned}
$$

According to the representation theorem, $\int_{X} f d^{d} \mu_{\left\langle u^{2}, v\right\rangle}-2 \int_{X} f u d \stackrel{d}{\mu}\langle u, v\rangle$ equals the right hand side of (2.13), and consequently $\int_{X} f d_{\mu}^{c}\left\langle u^{2}, v\right\rangle=2 \int_{X} f u d_{\mu}^{c}\langle u, v\rangle$ q.e.d.

Theorem 2.2 (derivation property of $\stackrel{c}{\mu}\langle u\rangle_{\langle u}$ ).

$$
d \stackrel{\mu}{\mu}\langle u v, w\rangle^{c}=\tilde{u} d_{\mu\langle v, w\rangle}^{c}+\tilde{v} d \tilde{\mu}_{\langle u, w\rangle}^{c} \quad \text { for } u, v, w \in F_{b} .
$$

Proof. This follows from Lemma 2.4 in the same way as in [6; Lemma 5.4.2].

q.e.d.

\section{A transformation by a multiplicative functional into a conser- vative process}

In $\S 2$, we have proved that $C_{0}$-regular Dirichlet form $E$ on $L^{2}(X ; m)$ can be represented as

$$
\begin{aligned}
E(u, v)=\frac{1}{2} \int_{X} d_{\mu}^{c}\langle u, v\rangle & +\int_{X x X-d}(\tilde{u}(x)-\tilde{u}(y))(\tilde{v}(x)-\tilde{v}(y)) J(d x, d y) \\
& +\int_{X} \tilde{u}(x) \tilde{v}(x) k(d x) .
\end{aligned}
$$

We introduce the space

$$
\chi=\left\{\begin{array}{l}
\rho=R_{a} g, \alpha>0, g \text { is a strictly positive function in } \\
C_{b} \cap L^{1}(m), \int_{X} \rho^{2} d m=1
\end{array}\right\}
$$

Take $\rho=R_{\alpha} g \in \chi$. Then, $\rho\left(X_{t}\right)-\rho\left(X_{0}\right)$ can be expressed as $M^{[\rho]}+N^{[\rho]}$ $=\stackrel{c}{M}^{[\rho]}+\stackrel{d}{M}^{[\rho]}+N^{[\rho]}$, because $\rho \in F$. We consider the transformation of $\boldsymbol{M}$ by the multiplicative functional 


$$
\begin{aligned}
L_{t}= & \exp \left(\int_{0}^{t} \frac{1}{\rho\left(X_{s-}\right)} d M_{s}^{[\rho]}-\frac{1}{2} \int_{0}^{t} \frac{1}{\rho^{2}\left(X_{s}\right)} d\left\langle\stackrel{c}{M}^{[\rho]}\right\rangle_{s}\right) \\
& \times \prod_{\substack{0<s \leq t \\
X_{s-\ddagger} \neq X_{s}}} \frac{\rho\left(X_{s}\right)}{\rho\left(X_{s-}\right)} \exp \left(-\left(\frac{\rho\left(X_{s}\right)}{\rho\left(X_{s-}\right)}\right)-1\right) I_{(t<\zeta]} .
\end{aligned}
$$

Denote by $\tilde{\boldsymbol{M}}$ the transformed Markov process. The transition function $\widetilde{P}_{t}$ of $\tilde{M}$ has the expression

$$
\tilde{P}_{t} g(x)=\boldsymbol{E}_{x}\left(L_{t} g\left(X_{t}\right)\right), \quad x \in X .
$$

Remark 3.1. $\quad L_{t}$ is a solution of Doléans-Dade equation ([2])

$$
Z_{t}-1=\int_{0}^{t} Z_{s-} \frac{1}{\rho\left(X_{s-}\right)} d M_{s}^{[\rho]}, \quad t<\zeta,
$$

and $L_{t}$ admits a simpler expression

$$
L_{t}=\frac{\rho\left(X_{t}\right)}{\rho\left(X_{0}\right)} \exp \left(-\int_{0}^{t} \frac{A \rho}{\rho}\left(X_{s}\right) d s\right) I_{(t<\zeta)}
$$

by virtue of Ito formula applied to the semi-martingale $\rho\left(X_{t}\right)$. Here, $A \rho=$ $\alpha \rho-g$.

Theorem 3.1. 1) $\quad \tilde{\boldsymbol{M}}$ is $\rho^{2} m$-symmetric.

2) Let $(\widetilde{E}, \widetilde{F})$ be the Dirichlet space generated by $\tilde{M}$, then $F \subset \widetilde{F}$ and for $u, v \in F$

$$
\begin{gathered}
\widetilde{E}(u, v)=\frac{1}{2} \int_{X} \rho^{2} d_{\tilde{\mu}}^{c}\langle u, v\rangle+\int_{X x X-d}(\tilde{u}(x)-\tilde{u}(y))(\tilde{v}(x)-\tilde{v}(y)) \\
\times \rho(x) \rho(y) J(d x, d y) .
\end{gathered}
$$

Proof. 1) Let $\left\{K_{n}\right\}_{n=1}^{\infty}$ be a compact nest satisfying $\inf _{x \in K^{\prime}} \rho(x) \geqq r_{n}>0$ and $\stackrel{0}{K}_{n}$ be a fine interior of $K_{n}$, and denote by $\boldsymbol{M}^{(n)}\left(\operatorname{resp} . \tilde{\boldsymbol{M}}^{(n)}\right)$ the part of $\boldsymbol{M}$ (resp. $\tilde{\boldsymbol{M}})$ on $\stackrel{0}{K}_{n}$.

For any $f, g \in \beta_{b}^{+}$

$$
\begin{aligned}
\left((\widetilde{P})_{t}^{(n)} g, f\right)_{\rho^{2} m} & =\left(\boldsymbol{E}^{(n)}\left(L_{t} g\left(X_{t}\right)\right), f\right)_{\rho^{2} m} \\
& =\left(\boldsymbol{E}^{(n)}\left(\frac{\rho\left(X_{t}\right)}{\rho\left(X_{0}\right)} \exp \left(-\int_{0}^{t} \frac{A \rho}{\rho}\left(X_{s}\right) d s\right) g\left(X_{t}\right)\right), f \rho^{2}\right)_{m} \\
& =\boldsymbol{E}_{m}^{(n)}\left(f\left(X_{0}\right) \rho\left(X_{0}\right) \exp \left(-\int_{0}^{t} \frac{A \rho}{\rho}\left(X_{s}\right) d s\right) g\left(X_{t}\right) \rho\left(X_{t}\right)\right) \\
& =\lim _{k \rightarrow \infty} \boldsymbol{E}_{m}^{(n)}\left(f\left(X_{0}\right) \rho\left(X_{0}\right) \prod_{p=0}^{k-1} \exp \left(-\frac{A \rho}{\rho}\left(X_{\frac{p}{k} t}\right) \frac{t}{k}\right) g\left(X_{t}\right) \rho\left(X_{t}\right)\right) .
\end{aligned}
$$

On account of symmetry of $\boldsymbol{M}^{(n)}$, the last expression equals 
$\lim _{k \rightarrow \infty} \boldsymbol{E}_{m}^{(n)}\left(g\left(X_{0}\right) \rho\left(X_{0}\right) \prod_{p=0}^{k-1} \exp \left(-\frac{A \rho}{\rho}\left(X_{\frac{p-1}{k}}\right) \frac{t}{k}\right) f\left(X_{t}\right) \rho\left(X_{t}\right)\right)$ by [6; Lemma 4.2.2]. Hence, we have

$$
\left((\widetilde{P})_{t}^{(n)} g, f\right)_{\rho^{2}}=\left(g,(\widetilde{P})_{t}^{(n)} f\right)_{\rho^{2} m}
$$

and by letting $n \rightarrow \infty$, we get equality

$$
\left((\widetilde{P})_{t} g, f\right)_{\rho^{2} m}=\left(g,(\widetilde{P})_{t} f\right)_{\rho^{2} m} .
$$

2) First of all, we prove this for $u=R_{1}^{(n)} g\left(g \in C_{0}\right)$. We let

$$
\begin{aligned}
& \left(u-E^{(n)}\left(L_{t} u\left(X_{t}\right)\right), u\right)_{\rho^{2} m} \\
& \quad=\left(u-P_{t}^{(n)} u, u\right)_{\rho^{2} m}-\left(\boldsymbol{E}^{(n)}\left(\left(L_{t}-1\right) u\left(X_{t}\right)\right), u\right)_{\rho^{2} m} \\
& \quad=(I)_{t}-(I I)_{t} .
\end{aligned}
$$

Since $L_{t}-1=\frac{1}{\rho\left(X_{0}\right)} \int_{0}^{t} \exp \left(-\int_{0}^{s} \frac{A \rho}{\rho}\left(X_{u}\right) d u\right) d M_{s}^{[\rho]}, t<\zeta$, by Remark 3.1, $(I I)_{t}$ equals

$$
\boldsymbol{E}_{u \rho m}\left(u\left(X_{t}\right) \int_{0}^{t} \exp \left(-\int_{0}^{s} \frac{A \rho}{\rho}\left(X_{u}\right) d u\right) d M_{s}^{[\rho]} ; t<\tau^{(n)}\right) .
$$

If we set

$$
(I I I)_{t}=\boldsymbol{E}_{u \rho m}\left(u\left(X_{t}\right) \int_{0}^{t \wedge \tau^{(n)}} \exp \left(-\int_{0}^{s} \frac{A \rho}{\rho}\left(X_{u}\right) d u\right) d M_{s}^{[\rho]} ; t \geqq \tau^{(n)}\right)
$$

then, by Schwarz inequality,

$$
\begin{aligned}
\frac{1}{t^{2}}(I I I)_{t}^{2} & \leqq \frac{1}{t} \boldsymbol{E}_{|u| \rho m}\left(\int_{0}^{t \wedge \tau^{(n)}} \exp \left(-2 \int_{0}^{s} \frac{A \rho}{\rho}\left(X_{u}\right) d u\right) d\left\langle M^{[\rho]}\right\rangle_{t}\right) \\
& \times \frac{1}{t} \boldsymbol{E}_{|u| \rho m}\left(u\left(X_{t}\right)^{2} ; t \geqq \tau^{(n)}\right) .
\end{aligned}
$$

But the first factor of the right hand side is not greater than

$$
\frac{1}{t} e^{c t} \boldsymbol{E}_{|u| \rho m}\left(\left\langle M^{[\rho]}\right\rangle_{t \wedge \tau}(m)\right) \leqq C^{\prime} \frac{1}{t} \boldsymbol{E}_{m}\left(\left\langle M^{[\rho]}\right\rangle_{t}\right) \leqq 2 C^{\prime} E(\rho, \rho),
$$

and the second factor equals

$$
\frac{1}{t}\left(|u| \rho, P_{t} u^{2}-u^{2}\right)_{m}-\frac{1}{t}\left(|u| \rho, P_{t}^{(n)} u^{2}-u^{2}\right)_{m} \underset{t \rightarrow 0}{\longrightarrow} E\left(|u| \rho, u^{2}\right)-E\left(|u| \rho, u^{2}\right)=0 .
$$

Hence we get

$$
\frac{1}{t}(I I I)_{t} \rightarrow 0 \quad(t \rightarrow 0)
$$


This implies

$$
\lim _{t \downarrow 0} \frac{1}{t}(I I)_{t}=\lim _{t \downarrow 0} \frac{1}{t} \boldsymbol{E}_{u \rho m}\left(u\left(X_{t}\right) \int_{0}^{t \wedge \tau^{(n)}} \exp \left(-\int_{0}^{s} \frac{A \rho}{\rho}\left(X_{u}\right) d u\right) d M_{s}^{[\rho]}\right),
$$

which in turn equals

$$
\begin{aligned}
& \lim _{t \downarrow 0} \frac{1}{t} \boldsymbol{E}_{u \rho m}\left(\left(u\left(X_{t}\right)-u\left(X_{0}\right)\right) \int_{0}^{t \wedge \tau^{(n)}} \exp \left(-\int_{0}^{s} \frac{A \rho}{\rho}\left(X_{u}\right) d u\right) d M_{s}^{[\rho]}\right) \\
= & \lim _{t \downarrow 0} \frac{1}{t} \boldsymbol{E}_{u \rho m}\left(\int_{0}^{t \wedge \tau^{(n)}} \exp \left(-\int_{0}^{s} \frac{A \rho}{\rho}\left(X_{u}\right) d u\right) d\left\langle M^{[u]}, M^{[\rho]}\right\rangle_{s}\right) .
\end{aligned}
$$

Noticing that there is a constant $N$ such that

$$
\left|\frac{\exp \left(-\int_{0}^{s} \frac{A \rho}{\rho}\left(X_{u}\right) d u\right)-1}{t}\right| \leqq N \quad \text { for } s \leqq t \wedge \tau^{(n)},
$$

we have

$$
\lim _{t \downarrow 0} \frac{1}{t}(I I)_{t}=\lim _{t \downarrow 0} \frac{1}{t} \boldsymbol{E}_{u \rho m}\left(\left\langle M^{[u]}, M^{[\rho]}\right\rangle_{t \wedge \tau}(n)\right)=\int_{X} u \rho d \mu_{\langle\rho, u\rangle} .
$$

Last equality holds by [6; Lemma 5.1.5].

On the other hand, we have

$$
\lim _{t \downarrow 0} \frac{1}{t}(I)_{t}=E\left(u, u \rho^{2}\right)
$$

From (3.6), (3.8) and (3.9), we have finally that $u \in \widetilde{F}$ and

$$
\widetilde{E}(u, u)=E\left(u, u \rho^{2}\right)-\int_{X} u \rho d \mu_{\langle\rho, u\rangle} .
$$

We can see that the right hand side of (3.10) equals the right hand side of (3.5). To see this, rewrite the right hand side of (3.10) as a sum of two terms $I$ and $I I$ where

$$
\begin{aligned}
& I=\frac{1}{2} \int_{X} d \dot{\mu}_{\left\langle u, u \rho^{2}\right\rangle}-\int_{X} u \rho d_{\mu}^{c}\langle\rho, u\rangle \\
& I I=\int_{X x X-d} w(x, y) J(d x, d y)
\end{aligned}
$$

with

$$
\begin{aligned}
w(x, y) & =(u(x)-u(y))\left(u(x) \rho^{2}(x)-u(y) \rho^{2}(y)\right) \\
& -2 u(x) \rho(x)(\rho(x)-\rho(y))(u(x)-u(y)) .
\end{aligned}
$$

Note that the contribution from the killing parts cancels out at this stage. By the derivation property, we easily see that 


$$
I=\frac{1}{2} \int_{X} \rho^{2} d_{\mu\langle u\rangle}^{c}
$$

On the other hand, since $w(x, y)-(u(x)-u(y))^{2} \rho(x) \rho(y)=(u(x)-u(y))$ $\times(\rho(x)-\rho(y)) u(y) \rho(y)-(u(x)-u(y))(\rho(x)-\rho(y)) u(x) \rho(x)$ and the measure $J$ is symmetric,

$$
I I=\int_{X x X-d}(u(x)-u(y))^{2} \rho(x) \rho(y) J(d x, d y) .
$$

We have proved (3.5) for $u=R_{1}^{(n)} g, g \in C_{0}$, and consequently $\widetilde{E}_{1}(u, u) \leqq$ $\|\rho\|_{\infty}^{2} E_{1}(u, u)$ for $u=R_{1}^{(u)} g, g \in C_{0}$. Now we can get the conclusion of Theorem 3.1 because $\lim _{n \rightarrow \infty} R_{1}^{(n)} g=R_{1} g$ in $E_{1}$-norm for $g \in C_{0}$ and $\left\{R_{1} g, g \in C_{0}\right\}$ is a dense subset of $F$.

q.e.d.

Lemma 3.1. If $B$ is a nearly Borel set and $v \in F_{b}$ satisfies $\inf _{x \in B}|v(x)|=\delta>0$, then $\frac{u}{v} \in F_{B}$ for $u \in F_{B}^{b}$.

Proof. Since

$$
\left|\frac{u(x)}{v(x)}\right| \leqq \frac{1}{\delta}|u(x)|,\left|\frac{u(x)}{v(x)}-\frac{u(y)}{v(y)}\right| \leqq \frac{2}{\delta}|u(x)-u(y)|+\frac{1}{\delta}|u(x) v(x)-u(y) v(y)|,
$$

and $u v \in F_{B}$, we can conclude $\frac{u}{v} \in F_{B}$.

q.e.d.

We may assume that $\tilde{\boldsymbol{M}}$ is a standard process on $X$ and has the elements $\Omega, X_{t}, \zeta$ in common with $\boldsymbol{M}$. We then have ([10])

$$
\tilde{\boldsymbol{P}}_{x}(\Lambda ; t<\zeta)=\boldsymbol{E}_{x}\left(L_{t} ; \Lambda\right), \quad \Lambda \in \beta_{t}, x \in X,
$$

where $\beta_{t}$ is the $\sigma$-field of $\Omega$ generated by $X_{s}, \mathrm{~s} \leqq t$. It follows from (3.11) that the notion of the exceptionality of sets and consequently the q.e. statements are the same for $\boldsymbol{M}$ and $\tilde{\boldsymbol{M}}$.

Theorem 3.2. $\quad \tilde{\boldsymbol{M}}$ is conservative: $\quad \tilde{\boldsymbol{P}}_{x}(\tilde{\zeta}<+\infty)=0$, q.e. $x \in X$.

Proof. It suffices to prove that $1 \in \widetilde{F}$ and $\widetilde{E}(1,1)=0$ ([7]). Let us put $h_{n}=\frac{R_{\alpha}^{(n)} g}{R_{\infty} g}$. By Theorem 3.1 and Lemma 3.1,

$$
h_{n} \in F_{K_{n}}^{0} \cap \widetilde{F}
$$

Since

$$
\begin{aligned}
& \left.d_{\mu\left\langle R_{\alpha}^{(n)} g-R_{\alpha}^{(m)} g\right\rangle}^{c}=d_{\mu\langle R a g}^{c}\left(h_{n}-h_{m}\right)\right\rangle \\
& =2\left(h_{n}-h_{m}\right) d_{\left.\mu_{\left\langle R_{\alpha \sigma} g\right.}^{c}\left(h_{n}-h_{m}\right), R \omega g\right\rangle}+\left(R_{\infty} g\right)^{2} d_{\left.\mu_{\left\langle h_{n}-h_{m}\right\rangle}^{c}\right\rangle} \\
& -\left(h_{n}-h_{m}\right)^{2} d_{\mu\langle R \sigma g\rangle}^{c},
\end{aligned}
$$


we have

$$
\begin{aligned}
\widetilde{E}^{(c)}\left(h_{n}-h_{m}, h_{n}-h_{m}\right) & =\frac{1}{2} \int_{X} d_{\mu}^{c}{ }_{\left\langle R_{\alpha}^{(n)} g-R_{\alpha}^{(m)} g\right\rangle}+\frac{1}{2} \int_{X}\left(h_{n}-h_{m}\right)^{2} d_{\mu}^{c}\langle R a g\rangle \\
& -\int_{X}\left(h_{n}-h_{m}\right) d_{\mu}^{c}\left\langle R_{a}^{(n)} g-R_{a}^{(m)} g, R_{a g}\right\rangle .
\end{aligned}
$$

The first and second terms of (3.13) clearly tend to zero by letting $n, m$ to infinite. Since

$$
\left|\int_{X}\left(h_{n}-h_{m}\right) d_{\mu}^{c}\left\langle R_{\alpha}^{(n)} g-R_{\alpha}^{(m)}\right)_{\left.g, R_{\alpha g}\right\rangle}\right| \leqq\left(\int_{X}\left(h_{n}-h_{m}\right)^{2} d \mu_{\left\langle R_{\alpha g}\right\rangle}^{c}\right)^{1 / 2}\left(\int_{X} d_{\mu}^{c}\left\langle_{\left\langle R_{\alpha}^{(n)} g-R_{\alpha}^{(m)} g\right\rangle}\right)^{1 / 2}\right.
$$

by Schwarz inequality, the third term also tends to zero.

On the other hand, we get

$$
\widetilde{E}^{(j)}\left(h_{n}-h_{m}, h_{n}-h_{m}\right)=\int_{X x X-d} \frac{\left(R_{\omega} g(x) R_{\alpha}^{n, m} g(y)-R_{a s} g(y) R_{\alpha}^{n, m} g(x)\right)^{2}}{R_{\infty} g(x) R_{\infty} g(y)} J(d x, d y)
$$

where $R_{\alpha}^{n, m} g=R_{\alpha}^{(n)} g-R_{\alpha}^{(m)} g$. Since $\left|R_{a} g(x) R_{\alpha}^{n, m} g(y)-R_{\infty} g(y) R_{\alpha}^{n, m} g(x)\right| \leqq R_{a} g(x)$ $\left|R_{a}^{n, m} g(y)-R_{a}^{n, m} g(x)\right|+\left|R_{\alpha}^{n, m} g(x)\right|\left|R_{a b} g(x)-R_{a} g(y)\right|$ and the left hand side is also dominated by $R_{\infty} g(y)\left|R_{\alpha}^{n, m} g(x)-R_{\alpha}^{n, m} g(y)\right|+\left|R_{\alpha}^{n, m} g(y)\right|\left|R_{\alpha} g(y)-R_{a} g(x)\right|$, it holds that

$$
\begin{aligned}
& \widetilde{E}^{(j)}\left(h_{n}-h_{m}, h_{n}-h_{m}\right) \leqq \int_{X x X-d}\left(R_{\alpha}^{n, m} g(x)-R_{a}^{n, m} g(y)\right)^{2} J(d x, d y) \\
& \quad+2 \int_{X x X-d}\left|h_{n}(x)-h_{m}(x)\right|\left|R_{\alpha}^{n, m} g(x)-R_{a}^{n, m} g(y)\right|\left|R_{\infty} g(x)-R_{a} g(y)\right| J(d x, d y) \\
& \quad+\int_{X x X-d}\left|h_{n}(x)-h_{m}(x)\right|\left|h_{n}(y)-h_{m}(y)\right|\left(R_{a} g(x)-R_{a} g(y)\right)^{2} J(d x, d y) .
\end{aligned}
$$

The first term of the right hand side clearly tends to zero by letting $n, m$ to infinite and the third term tends to zero by the bounded convergence theorem. Finally we see the second term also tends to zero since it is dominated by

$$
\begin{aligned}
2\left(\int_{X x X-d}(\right. & \left.\left(h_{n}(x)-h_{m}(x)\right)^{2}\left(R_{a x} g(x)-R_{\infty} g(y)\right)^{2} J(d x, d y)\right)^{1 / 2} \\
& \times\left(\int_{X x X-d}\left(R_{\infty}^{n, m} g(x)-R_{\alpha}^{n, m} g(y)\right)^{2} J(d x, d y)\right)^{1 / 2},
\end{aligned}
$$

and this means $\widetilde{E}^{(j)}\left(h_{n}-h_{m}, h_{n}-h_{m}\right) \rightarrow 0(n, m \rightarrow \infty)$. Therefore, it holds that $\widetilde{E_{1}}\left(h_{n}-h_{m}, h_{n}-h_{m}\right) \rightarrow 0(n, m \rightarrow \infty)$. Moreover, because $h_{n} \rightarrow 1(n \rightarrow \infty), 1 \in \widetilde{F}$.

Next we get $\lim _{n \rightarrow \infty} \widetilde{E}\left(h_{n}, h_{n}\right)=0$, because

$$
\begin{aligned}
& \int_{X} \rho^{2} d_{\mu_{\left\langle h_{n}\right\rangle}^{c}}^{c}=\int_{X} d_{\left.\mu_{\left\langle R_{\alpha}\right.}^{(n)} g\right\rangle}^{c}+\int_{X} h_{n}^{2} d_{\left.\mu_{\langle R a g}\right\rangle}^{c}-2 \int_{X} h_{n} d_{\left.\left.\mu_{\left\langle R_{\alpha}(n)\right.}^{c}\right)_{g, R g}\right\rangle} \\
& \underset{n \rightarrow \infty}{\longrightarrow} 2 \int_{X} d_{\mu_{\langle R a g\rangle}^{\tau c}}^{c}-2 \int_{X} d \dot{\mu}_{\langle R \alpha g\rangle}^{c}=0,
\end{aligned}
$$


and

$$
\begin{aligned}
\int_{X x X-d}( & \left.h_{n}(x)-h_{n}(y)\right)^{2} J(d x, d y)=\int_{X x X-d}\left(R_{\infty}^{(n)} g(x)-R_{\infty}^{(n)} g(y)\right)^{2} J(d x, d y) \\
& -2 \int_{X x X-d} h_{n}(x)\left(R_{\infty} g(x)-R_{\infty} g(y)\right)\left(R_{a}^{(n)} g(x)-R_{\infty}^{(n)} g(y)\right) J(d x, d y) \\
& +\int_{X x X-d} h_{n}(x) h_{n}(y)\left(R_{\infty} g(x)-R_{\infty} g(y)\right)^{2} J(d x, d y) \\
& \underset{n \rightarrow \infty}{\longrightarrow} \int_{X x X-d}\left(R_{\infty} g(x)-R_{\infty} g(y)\right)^{2} J(d x, d y) \\
& -2 \int_{X x X-d}\left(R_{\infty} g(x)-R_{\infty} g(y)\right)^{2} J(d x, d y)=0 .
\end{aligned}
$$

This means $\widetilde{E}(1,1)=0$. Therefore, we conclude that $\tilde{M}$ is conservative. q.e.d.

By virtue of (3.11) and Theorem 3.2, it holds that

$$
\boldsymbol{P}_{x}(\Lambda ; t<\zeta)=\tilde{\boldsymbol{E}}_{x}\left(\frac{\rho\left(X_{0}\right)}{\rho\left(X_{t}\right)} \exp \left(\int_{0}^{t} \frac{A \rho}{\rho}\left(X_{s}\right) d s\right) ; \Lambda\right) \quad \text { q.e. } x \in X \Lambda \in \beta_{t}
$$

This formula will be used in the next section.

\section{A lower estimate related to the Donsker-Varadhan theory}

The Dirichlet space $(E, F)$ is called irreducible if any $P_{t}$-invariant set is trivial, namely, a Borel set $A \subset X$ satisfies either $m(A)=0$ or $m(X-A)=0$ whenever $P_{t}\left(I_{A} u\right)=I_{A} P_{t} u$ for any $u \in \beta_{b}^{+}$and $t>0$. Denote by $\mathscr{M}$ the space of probability measures equipped with the weak topology and let $L(t, \omega, A)=$ $\int_{0}^{t} I_{A}\left(X_{s}\right) d s$

Proposition 4.1. If $(E, F)$ is irreducible, then for $\rho \in \chi$

$$
\varliminf_{t \rightarrow \infty} \frac{1}{t} \boldsymbol{P}_{x}\left(L(t, \omega, \cdot) \in N_{\rho^{2}}, t<\zeta\right) \geqq-E(\rho, \rho) \quad \text { q.e. } x \in X
$$

where $N_{\rho^{2}}$ is any neighborhood of $\rho^{2} m \in \mathscr{M}$.

Proof. By (3.15),

$$
\boldsymbol{P}_{x}\left(L(t, \omega, \cdot) \in N_{\rho^{2}}, t<\zeta\right)=\tilde{\boldsymbol{E}}_{x}\left(\frac{\rho\left(X_{0}\right)}{\rho\left(X_{t}\right)} \exp \left(\int_{0}^{t} \frac{A \rho}{\rho}\left(X_{s}\right) d s\right) ; L(t, \omega, \cdot) \in N_{\rho^{2}}\right)
$$
q.e. $x \in X$.

We set

$$
S(t, \varepsilon)=\left\{\omega \in \Omega ;\left|\int_{X} \frac{A \rho}{\rho}(y) L(t, \omega, d y)-\int_{X} \rho A \rho d m\right|<\varepsilon\right\}
$$




$$
S^{\prime}(t, \varepsilon)=\left\{\omega \in \Omega ; L(t, \omega, \cdot) \in N_{\rho}^{2}\right\} \cap S(t, \varepsilon)
$$

then, the right hand side is greater than

$$
\begin{gathered}
\exp \left(t\left(\int_{X} \rho A \rho d m-\varepsilon\right)\right) \times \tilde{\boldsymbol{E}}_{x}\left(\frac{\rho\left(X_{0}\right)}{\rho\left(X_{t}\right)} ; S^{\prime}(t, \varepsilon)\right) \geqq \exp \left(t\left(\int_{X} \rho A \rho d m-\varepsilon\right)\right) \\
\times \frac{\rho(x)}{\|\rho\|_{\infty}}\left(1-\tilde{\boldsymbol{P}}_{x}\left(\Omega-S^{\prime}(t, \varepsilon)\right)\right) .
\end{gathered}
$$

Now, the irreducibility of $(E, F)$ implies the same property of $(\widetilde{E}, \widetilde{F})$ because $L_{t}$ is positive until the life time $\zeta$. Since $\tilde{\boldsymbol{M}}$ is conservative with invariant measure $\rho^{2} d m$ by Theorem $3.2, \tilde{\boldsymbol{M}}$ is ergodic ([9]) and

$$
\tilde{\boldsymbol{P}}_{\rho^{2} m}\left(\lim _{t \rightarrow \infty} \frac{1}{t} \int_{0}^{t} \frac{A \rho}{\rho}\left(X_{s}\right) d s=\int_{X} \rho A \rho d m\right)=1 .
$$

If we denote by $\Lambda$ the event inside the braces of $(4.3)$, we get $\tilde{\boldsymbol{P}}_{x}(\Lambda)=1, \tilde{\boldsymbol{M}}$-q.e. by noticing that $\tilde{\boldsymbol{P}}_{x}(\Lambda)$ is an excessive function. By virtue of the remark made before Theorem 3.2, we then have $\varliminf_{t \rightarrow \infty} \tilde{\boldsymbol{P}}_{x}\left(\Omega-S^{\prime}(t, \varepsilon)\right)=0$, q.e. Therefore

$$
\varliminf_{t \rightarrow \infty} \frac{1}{t} \log \boldsymbol{P}_{x}\left(L(t, \omega, \cdot) \in N_{\rho^{2}} ; t<\zeta\right) \geqq \int_{X} \rho A \rho d m-\varepsilon, \text { q.e. }
$$

As $\varepsilon$ is arbitrary and $\int_{X} \rho A \rho d m=-E(\rho, \rho)$, we arrive at Proposition 4.1. q.e.d.

From now we require the following assumption.

Assumption 4.1. There exists a sequence $\mathcal{G}=\left\{G_{n}\right\}_{n=1}^{\infty}$ of increasing relatively compact open sets satisfying: 1) $\left.\bigcup_{n} G_{n}=X, 2\right)\left(E_{G_{n}}, F_{G_{n}}\right)$ is irreducible.

A comparison theorem concerning the irreducibility of local Dirichlet forms has been given in [8]; if $E^{(1)}$ and $E^{(2)}$ are such forms, $E^{(1)}$ is dominating $E^{(2)}$ on a common core and $E^{(2)}$ is irreducible, then $E^{(1)}$ is also irreducible. From this, we can show that the first two examples in $\S 1$ satisfy Assumption 4.1. The irreducibility of the third example in $\S 1$ follows from the relation between $J$ and the Lévy system stated in $\S 2$.

Let us introduce the following space

$$
\tilde{\chi}=\left\{\rho ; \rho=R_{\alpha}^{G_{n}} g, \alpha>0, g>0, g \in C_{b}, \int_{X} \rho^{2} d m=1, n=1,2, \cdots\right\}
$$

and let $\Phi$ be a function on $\mathscr{M}$ such that $\Phi\left(\mu_{n}\right) \rightarrow \Phi(\mu)$ whenever $\mu_{n} \in \mathscr{M}$ converge weakly to $\mu$ and the support of $\mu_{n}$ is contained in some compact set. We further assume that $\Phi(\mu) \neq-\infty$ for any $\mu \in \mathscr{M}$.

Lemma 4.1. It holds that 


$$
\varliminf_{t \rightarrow \infty} \frac{1}{t} \log \boldsymbol{E}_{x}\left(e^{-t \Phi(L(t, \omega, \cdot))}\right) \geqq-\inf _{\rho \in \tilde{x}}\left[\Phi\left(\rho^{2}\right)+E(\rho, \rho)\right] \quad \text { q.e. }
$$

Proof. Take $\rho=R_{\infty}^{G_{n}} g \in \tilde{\chi}$. Since $\left(E_{G_{n}}, F_{G_{n}}\right)$ is an irreducible $C_{0}$-regular Dirichlet space, it follows from Proposition 4.1

$$
\varliminf_{t \rightarrow \infty} \frac{1}{t} \log \boldsymbol{P}_{x}\left(L(t, \omega, \cdot) \in N_{\rho^{2}}, t<\zeta\right) \geqq-E(\rho, \rho) \quad \text { q.e. }
$$

Therefore, this theorem can be shown by the same method as [3].

q.e.d.

\section{Lemma 4.2. It holds that}

$$
\inf _{\rho \in \tilde{x}}\left[\Phi\left(\rho^{2}\right)+E(\rho, \rho)\right] \leqq \inf _{\rho \in \sigma}\left[\Phi\left(\rho^{2}\right)+E(\rho, \rho)\right]
$$

where $U=\left\{\rho \in F \cap C_{0}^{+} ; \int_{X} \rho^{2} d m=1\right\}$.

Proof. For any $\rho \in U$, take $G_{n} \in \mathcal{G}$ such that $\operatorname{Supp}[\rho m] \subset G_{n}$. Since $\alpha R_{\alpha}^{G_{n}} \rho \rightarrow \rho(\alpha \rightarrow \infty)$ in $E_{1}$ and $R_{\alpha}^{G_{n}}(\rho \vee \varepsilon) \rightarrow R_{\alpha}^{G_{n}} \rho(\varepsilon \rightarrow 0)$ in $E_{1}$, there exists a sequence $\left\{\rho_{p}=R_{\alpha}^{G_{n}} g_{p} ; \alpha>0, g_{p}>0, g_{p} \in C_{b}\right\}_{p=1}^{\infty}$ such that $\rho_{p} \rightarrow \rho(p \rightarrow \infty)$ in $E_{1}$. Therefore, $\frac{\rho_{p}}{\left\|\rho_{p}\right\|_{L^{2}}} \in \tilde{\chi} \rightarrow \rho(p \rightarrow \infty)$ in $E_{1}$, and we get (4.6) in view of the property of $\Phi$.

q.e.d.

\section{Lemma 4.3. It holds that}

$$
\inf _{\rho \in U}\left[\Phi\left(\rho^{2}\right)+E(\rho, \rho)\right]=\inf _{\rho \in V}\left[\Phi\left(\rho^{2}\right)+E(\rho, \rho)\right]
$$

where $V=\left\{\rho \in F ; \operatorname{Supp}[\rho m]\right.$ is compact. $\left.\int_{X} \rho^{2} d m=1\right\}$.

Proof. For $\rho \in V$, we take the relatively compact open set such that Supp $[\rho m] \subset G$. Since $\left(E_{G}, F_{G}\right)$ is a regular Dirichlet space, there exists a sequence $\left\{\rho_{n}\right\} \subset F_{G} \cap C_{0}$ such that $\rho_{n} \rightarrow \rho$ in $E_{1}$. Therefore, noting that $E\left(\left|\rho_{n}\right|,\left|\rho_{n}\right|\right) \leqq E\left(\rho_{n}, \rho_{n}\right)$, we see that the left hand side is not greater than the right in the same way as Lemma 4.2.

q.e.d.

Theorem 4.1. It holds that

$$
\varliminf_{t \rightarrow \infty} \frac{1}{t} \log E_{x}\left(e^{-t \Phi(L(t, \omega, \cdot))}\right) \geqq-\inf _{\rho \in V}\left[\Phi\left(\rho^{2}\right)+E(\rho, \rho)\right] \quad \text { q.e. }
$$

Proof. By virtue of Lemma 4.2 and Lemma 4.3, we have

$$
\inf _{\rho \in \tilde{\mathbb{X}}}\left[\Phi\left(\rho^{2}\right)+E(\rho, \rho)\right]=\inf _{\rho \in V}\left[\Phi\left(\rho^{2}\right)+E(\rho, \rho)\right] .
$$

Hence this theorem is clear by Lemma 4.1.

q.e.d. 
The above proof shows that, in equality (4.9), we can replace the space $V$ by a smaller one $V \cap D$ where $D$ is any core of $F$ satisfying the conditions of [8; pp. 198].

\section{References}

[1] C. Dellacherie and P.A. Meyer: Probabilités et potentiel, Hermann, Paris, 1980.

[2] C. Doléans-Dade: Quelques applications de la formule de changement de variables pour les semimartingales, Z. Wahrsch. Verw. Gebiete 16 (1970), 181-194.

[3] M.D. Donsker and S.R.S. Varadhan: Asymptotic evaluation of certain Wiener integrals for large time, in Functional Integration and its Application, (A.M. Arthur, ed.) London Clarendon Press, Oxford, 1975, 15-33.

[4] M.D. Donsker and S.R.S. Varadhan: Asymptotic evaluation of certain Markov process expectations for large time I, Comm. Pure. Appl. Math. 28 (1975), 1-47.

[5] M.D. Donsker and S.R.S. Varadhan: Asymptotic evaluation of certain Markov process expectations for large time III, Comm. Pure Appl. Math. 29 (1976), 389461.

[6] M. Fukushima: Dirichlet forms and Markov processes, North-Holland and Kodansha, 1980.

[7] M. Fukushima: On a stochastic calculus related to Dirichlet forms and distorted Brownian motion, Physics Report 502, North-Holland, 1981.

[8] M. Fukushima: Markov processes and functional analysis, Proceedings of International Mathematical Conference, Singapore, North-Holland, 1982.

[9] M. Fukushima: $A$ note on irreducibility and ergodicity of symmetric Markov process, Proceedings, Marseille, Lecture Note in Physics 173, Springer, 1982.

[10] H. Kunita and T. Watanabe: Note on transformations of Markov processes connected with multiplicative functionals, Mem. Fac. Sci. Kyushu Univ. Ser. A 17 (1963), 181-191.

[11] Y. LeJan: Mesures associées a une forme de Dirichlet, Application, Bull. Soc. Math. France 106 (1978), 61-112.

[12] M. Tomisaki: Dirichlet forms associated with direct product diffusion processes, Lecture Notes in Math. 923, Springer, 1981.

Masatoshi Fukushima

Department of Mathematics

College of General Education

Osaka University

Toyonaka, Osaka, Japan

Masayoshi Takeda

Department of Mathematics

Osaka University

Toyonaka, Osaka, Japan 